\title{
Fluctuations de pression induites par les singularités d'écoulement dans les circuits industriels
}

\section{Pressure fluctuations caused in industrial circuits by singular flow}

\author{
René-Jean Gibert et Bernard Villard \\ Commissariat à l'Energie Atomique - Centre d'Etudes Nucléaires de Saclay \\ D.E.M.T. \\ B.P. $n^{\circ} 2-91190$ Gif-sur-Yvette
}

\section{Introduction}

Les circuits parcourus par des fluides sont le siège de phénomènes vibratoires.

Ces phénomènes sont, dans la majorité des cas, engendrés par les fluctuations de la pression du fluide aux parois qui créent sur les structures mécaniques des efforts fluctuants.

Ces efforts excitent les résonances de ces structures et peuvent parfois occasionner des déplacements et des contraintes fluctuantes telles que des ruptures (par fatigue, par chocs, etc...) se produisent.

Pour qu'une excitation mécanique des structures soit importante, il faut bien sûr que l'amplitude des fluctuations de pression soit grande, mais aussi que les longueurs de corrélation de ces fluctuations ne soient pas trop petites vis-à-vis des dimensions caractéristiques de ces structures.

Il apparaît donc que la turbulence établie dans un tube droit parcouru par un fluide, qui est un phénomène à très petite échelle et d'intensité assez faible, aura un effet négligeable sur les structures. En revanche, des fluctuations à plus grande longueur de corrélation et beaucoup plus intenses peuvent prendre naissance en certains points particuliers du circuit, que nous appellerons d'une façon générale, singularités.

$\mathrm{Au}$ niveau d'une singularité, l'écoulement turbulent sera qualifié d'instationnaire, par opposition avec le régime quasi-stationnaire, de la turbulence établie.

Un ensemble d'études a été développé au CEA/DEMT concernant les principales singularités simples rencontrées dans un circuit industriel : élargissements brusques ou progressifs, jonctions d'écoulements en $T$, coudes, vannes, etc...

Un ensemble cohérent de résultats théoriques et expérimentaux a été dégagé dont nous présentons ici les principales caractéristiques illustrées de quelques exemples.

Pour plus de détail, nous renvoyons le lecteur à la référence [9] où l'ensemble des résultats obtenus est consigné sous forme d'un catalogue directement utilisable au niveau d'un projet d'installation industrielle, par exemple.

Distinction entre les deux aspects de l'effet de singularité dans un circuit

Soit un circuit contenant une singularité. Les caractéristiques spectrales des fluctuations de pression varient sensiblement suivant la région du circuit dans laquelle on effectue la mesure :

1) A l'aval immédiat de la singularité, l'écoulement est très perturbé. Il s'agit d'une turbulence à grande échelle, son effet sur les structures peut être important.

Les spectres d'autocorrélation des fluctuations de pression sont en général à large bande, les gammes de fréquences caractéristiques évoluent proportionnellement à la vitesse "moyenne" de l'écoulement. Les fonctions de cohérence entre deux signaux issus de deux capteurs évoluent avec la distance entre ces derniers.

2) Dans le reste du circuit (à l'amont et loin à l'aval de la singularité), la turbulence de l'écoulement est d'intensité faible et l'on observe essentiellement un phénomène de nature acoustique : les spectres d'autocorrélation des fluctuations de pression comportent des pics dont la fréquence n'évolue quasiment pas avec la vitesse de l'écoulement. Il s'agit des pics de résonance acoustique du circuit. De plus les fonctions de cohérence sont égales à 1 dans de larges plages de fréquence (quelle que soit la distance entre les capteurs). 
Ceci veut dire que les signaux mesurés sont obtenus à partir d'une même source aléatoire "vue à travers la fonction de transfert acoustique du circuit".

Les singularités devront donc être caractérisées suivant deux points de vue :

- Du point de vue "local" : il s'agit d'analyser le phénomène décrit au paragraphe 1, qui ne dépend que de la géométrie et de l'écoulement au voisinage de la singularité.

- Du point de vue "acoustique" : il s'agit d'analyser le phénomène décrit au paragraphe 2 et plus particulièrement la source acoustique associée qui, comme le phénomène local, ne dépend que de la géométrie et de l'écoulement au voisinage de la singularité.

Caractéristiques générales des fluctuations locales engendrées par les singularités

Les considérations développées dans ce paragraphe sont basées sur des résultats expérimentaux obtenus sur des essais en eau et en air atmosphérique.

\section{A. Importance de la connaissance de certaines grandeurs caractéristiques de l'écoulement permanent}

Il est important de connaître certaines caractéristiques de l'écoulement permanent au niveau de la singularité. En effet, comme nous l'illustrerons plus loin, on peut former, à partir de grandeurs permanentes bien choisies, des variables et fonctions adimensionnelles telles que spectres, fréquences adimensionnelles, etc. . . Les caractéristiques spectrales et interspectrales des fluctuations peuvent alors s'exprimer en fonction de ces variables adimensionnelles suivant des lois standard où n'intervient que la nature de la singularité (il est à remarquer d'ailleurs que souvent d'une singularité à une autre les lois adimensionnelles sont fort voisines). Ces lois sont quasi indépendantes des nombres de Reynolds et de Mach de l'écoulement (il est cependant à remarquer que les expériences qui servent de base à ce rapport ont balayé une gamme de Reynolds de $0,5 \times 10^{5}$ à $2 \times 10^{6}$ et une gamme de nombres de Mach de 0,01 à 0,5 environ).

Les principales grandeurs permanentes dont on a besoin sont :

a) Une pression de référence $\Delta P$ à laquelle nous rapportons les fluctuations de pression. Cette pression est souvent la différence des pressions statiques dans deux zones particulières de la singularité.

b) Une vitesse de référence $V$ : on prend souvent la vitesse moyenne la plus grande dans la singularité.

c) Une ou plusieurs dimensions caractéristiques auxquelles nous rapportons les variables d'espace intervenant par exemple dans les lois de corrélation et qui interviennent également avec la vitesse $V$ dans la détermination des fréquences caractéristiques des fluctuations.

Pour une singularité donnée, le choix des bonnes grandeurs permanentes est à rapprocher du. choix d'un bon modèle représentant le mécanisme de l'instabilité dans la singularité.

Pour les singularités que nous avons analysées, l'expé- rience a permis de comprendre ces mécanismes et donc de choisir de bonnes grandeurs permanentes.

En ce qui concerne les autres singularités, en l'absence de toute donnée expérimentale, l'extrapolation de certains mécanismes du même type permet vraisemblablement une estimation honnête des caractéristiques des fluctuations.

\section{B. Analyse des caractéristiques générales des grandeurs statistiques associées aux fluctuations locales}

\section{1) Ecart type (valeur quadratique moyenne) des fluc- tuations}

C'est la grandeur statistique la plus simple associée aux fluctuations. Elle mesure "l'intensité". Cet écarttype est rapporté à la pression de référence $\Delta P$.

$\sigma / \Delta P$ est une fonction de la distance du point de mesure à la singularité et passe par un maximum d'environ 0,1 à 0,2 pour les singularités à décollement franc (élargissement brusque, réunions d'écoulements...). Ceci signifie que les fluctuations "crêtes à crêtes" de la pression sont de l'ordre de $\Delta P$. Le $\Delta P$ choisi correspond alors à la différence des pressions extrêmes entre lesquelles évolue la pression mesurée dans la singularité.

Pour les singularités du type diffuseur à $7^{\circ}$ où n'y a pas de décollement franc, $(\sigma / \Delta P)_{\max }$ est en général plus faible.

\section{2) Spectres d'autocorrélation des fluctuations}

A partir des grandeurs de référence, on définit une fréquence adimensionnelle ou nombre de Strouhal :

$$
\bar{s}=\frac{\nu \Delta}{V} \quad(\nu \text { étant la fréquence })
$$

et une densité spectrale adimensionnelle :

$$
\mathscr{S}(\bar{s})=\frac{\mathscr{S}(\nu)}{\Delta P^{2}} \frac{V}{\Delta} \quad \mathscr{S}(\nu) \text { étant la densité spectrale) }
$$

L'allure de $\mathscr{J}(s)$ est décroissante comme pour les spectres de turbulence. Une forme de type "Dryden" les représente en général assez bien :

$$
\widetilde{\oiint}(s)=\frac{K}{1+\left(s / s_{c}\right)^{2}} \quad \text { avec } \quad K=\left(\frac{\sigma}{\Delta p}\right)^{2} \frac{2 s_{c}}{\pi}
$$

$s_{c}$ est appelé nombre de Strouhal de coupure. $S_{c}$ est de l'ordre de 0,2 à 0,5 dans la zone où les fluctuations sont maximum.

\section{3) Spectres d'intercorrélation des fluctuations}

Nous utilisons pour étudier la corrélation spatiale des fluctuations de pression, la fonction de cohérence et la fonction phase. La fonction de cohérence est le rapport du module du spectre d'intercorrélation de deux signaux et de la racine carrée du produit des spectres d'autocorrélation de chacun des deux signaux.

La fonction phase est la phase du spectre d'intercorrélation.

La fonction de cohérence, tracée en fonction du nombre de Strouhal $s$ défini précédemment, présente en 
général un plateau limité supérieurement par un nombre de Strouhal voisin du nombre de Strouhal de coupure $s_{c}$.

Le niveau $C$ de ce plateau est généralement fonction exponentielle de la distance relative des deux capteurs que l'on corrèle : $C=e^{-\Delta 1 / \lambda} . \lambda$ est la largeur de corrélation des fluctuations et est du même ordre de grandeur que les dimensions caractéristiques de la singularité. On peut définir une longueur de corrélation longitudinale ou transversale suivant que les capteurs corrélés sont situés longitudinalement ou transversalement par rapport à l'écoulement moyen.

La fonction phase longitudinale, tracée en fonction de $s$ se présente sous la forme d'une droite qui est l'image d'un phénomène de transport des fluctuations à une vitesse $V_{t}$ telle que $\frac{V_{t}}{V}=\propto . \propto$ peut être déduit de la pente de la droite.

En général $\propto$ est de l'ordre de 0,7 à 0,9 .

Transversalement, les fluctuations sont en général en phase.

Caractéristiques générales des sources acoustiques associées aux singularités

\section{A. Mise en évidence théorique d'une source acoustique associée à une singularité}

Les travaux de Lighthill [réf. 1] ont montré les effets acoustiques d'un champ turbulent dans un espace libre tridimensionnel.

De même dans les tuyauteries, les zones très turbulentes, donc les singularités, constituent des sources acoustiques excitant l'ensemble du circuit.

La différence avec la théorie de Lighthill est le caractère interne et monodimensionnel des sources acoustiques associées aux singularités.

Nous avons étudié au chapitre précédent les caractéristiques générales des fluctuations locales engendrées par les singularités dans un circuit. Nous avons en particulier caractérisé leur spectre par un nombre de Strouhal de coupures $s_{c}$ au maximum égal à 0,5 . L'essentiel de l'énergie de la fluctuation se situe en-dessous de $s_{c}$.

Or, si l'on compare la longueur d'onde acoustique à la fréquence $\nu_{c}\left(s_{c}=\nu_{c} \Delta / V\right)$ a la dimension caractéristique de la singularité nous avons :

$$
\frac{\lambda_{c}}{\Delta}=\frac{1}{s_{c} M}
$$

( $M$ étant le nombre de Mach de l'écoulement).

Or le nombre de Mach dans les installations industrielles est en général petit devant 1 , la longueur d'onde des fluctuations générées par la singularité est donc grande vis-à-vis des dimensions de cette dernière. C'est-àdire que l'on se situe bien en-dessous de la fréquence de coupure acoustique des tubes constituant le circuit.

La propagation acoustique dans le circuit ne peut donc se faire que par ondes planes.

D'autre part la plupart des singularités se situent dans un tube (élargissements brusques, diffuseurs, coudes, etc...). Le volume d'écoulement perturbé a donc une entrée et une sortie. Le problème acoustique devient donc complètement monodimensionnel et nous montrons [réf. 6] que la singularité peut alors être caractérisée acoustiquement par une discontinuité $\Delta p(t)$ de la pression acoustique (p représentant alors la pression fluctuante acoustique en phase dans une section droite des tubes du circuit) et une discontinuité $\Delta q(t) d u$ débit masse acoustique.

Ces deux discontinuités sont des fonctions aléatoires du temps que l'on caractérise par leur spectre d'autocorrélation.

Une analyse dimensionnelle montre que les spectres adimensionnels $\widetilde{\dddot{H}^{\prime}} \Delta q(s)$ et $\widetilde{\oiint_{1}}(s)$ des fonctions $\Delta q(t)$ et $\Delta p(t)$, peuvent être mis sous la forme:

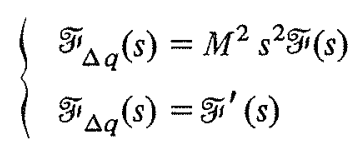

$M$ étant le nombre de Mach de référence de l'écoulement, $\mathscr{J}(s)$ et $\mathscr{H}^{\prime}(s)$ sont deux spectres adimensionnels caractéristiques liés aux fluctuations turbulentes dans la singularité, donc d'allure décroissante en $s$ (de type Dryden par exemple), Le graphique ci-après montre l'allure de $\mathscr{F}_{\Delta q}(s)$ et $\mathscr{\Im}_{\Delta p}(s)$ et en particulier qu'aux très bas nombres de Strouhal la singularité se comporte comme une discontinuité de pression acoustique et que pour des nombres de Strouhal plus élevés elle se comporte, si le nombre de Mach n'est pas trop petit (pratiquement $>0,2-0,3)$, comme une discontinuité de débit acoustique.

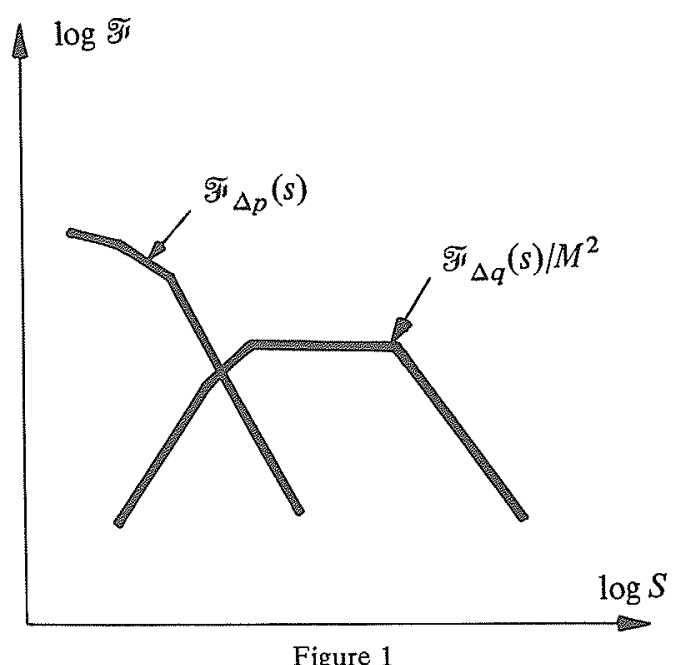

D'autre part, nous voyons que l'écart-type de la discontinuité $\Delta q(t)$ évolue avec la vitesse de référence de l'écoulement dans la singularité en $V^{3}(M \Delta P)$ alors que la discontinuité $\Delta p(t)$ évolue comme les fluctuations locales, c'est-à-dire en $V^{2}(\Delta P)$.

L'importance de la discontinuité $\Delta q(t)$ est donc d'autant plus grande que la vitesse de l'écoulement est élevée.

Cependant, dans la plupart des cas industriels, les nombres de Mach de l'écoulement sont faibles (surtout dans les écoulements de liquides) et l'on s'intéresse en général aux basses fréquences. C'est donc la discontinuité $\Delta p(t)$ qui est la plus importante. 


\section{B. Considérations sur la méthode de détermination expé- rimentale des sources acoustiques associées aux sin- gularités}

L'estimation des discontinuités de débit et pression acoustique associées à une singularité est théoriquement possible à partir des fluctuations locales. Les expressions théoriques sont explicitées à la réf. [6]. Cependant il faut pour cela connaitre le spectre d'intercorrélation des pressions et vitesses fluctuantes en tout point du volume de la singularité. Ceci est en général fort lourd.

$\mathrm{Si}$ donc ces formules peuvent être utiles pour obtenir un ordre de grandeur des sources acoustiques, la seule façon précise d'obtenir ces dernières est de faire une mesure acoustique directe.

Pour cela, on place la singularité à étudier dans un circuit et l'on mesure les fluctuations de pression engendrées soit à l'amont de la singularité, soit loin à l'aval. L'interprétation de ces mesures acoustiques pose un certain nombre de problèmes :

1) Il faut tout d'abord que dans le circuit expérimental la singularité soit l'unique source d'excitation acoustique du circuit

2) On ne mesure pas la source acoustique mais la réponse du circuit à cette source. Il est donc nécessaire de connaître parfaitement la fonction de transfert acoustique du circuit pour pouvoir déterminer correctement à partir des spectres des fluctuations de pression acoustique dans le circuit, le spectre de la source.

Pour cela il faut que les conditions aux limites du circuit soient acoustiquement bien définies et il faut en général disposer d'un code de calcul [voir réf. 7].

3) Il faut également que la fonction de transfert soit favorable dans la zone du circuit où l'on effectue la mesure. C'est-à-dire que son module ne soit pas trop petit devant 1 , que les résonances du circuit ne soient pas trop serrées dans la gamme de fréquence étudiée, etc...

4) Il faut enfin prendre un certain nombre de précautions pour que l'écoulement dans la singularité ne soit pas altéré (prévoir une certaine longueur de tube droit à l'aval et à l'amont de la singularité, étudier l'éventualité de couplages entre les fluctuations acoustiques et l'écoulement perturbé, etc...).

On pourra par exemple consulter la référence [1] qui décrit en détail de telles études expérimentales.

\section{Caractéristiques générales des fonctions $\Delta p(t)$ et $\Delta q(t)$}

\section{1) Discontinuité $\Delta p(t)$}

Nous avons déjà vu que le spectre $\$ \Delta p(\nu)$ de $\Delta p(t)$ pouvait être tracé en coordonnées adimensionnelles de la même façon que les spectres des fluctuations de pression locales. Nous choisirons en général les mêmes coordonnées adimensionnelles : le nombre de Strouhal $s=\nu \Delta / V$ et la densité spectrale adimensionnelle :

$$
\widetilde{\mathscr{A}}_{\Delta p}(s)=\frac{\mathscr{S}_{\Delta p}(\nu)}{\Delta p^{2}} \frac{V}{\Delta}
$$

Nous avons vu qu'alors अु ${ }_{\Delta \rho}(s)$ est indépendant du débit dans le circuit et que son allure est du type "tur. bulence". On peut définir comme pour les fluctuations locales, un nombre de Strouhal de coupure $s_{c}$ qui est en général plus petit que celui des fluctuations locales (souvent de l'ordre de 0,01).

L'écart type adimensionnel $\sigma / \Delta P$ est en général de l'ordre de $10^{-2}$ (1/10ème des fluctuations locales).

\section{2) Discontinuité $\Delta q(t)$}

Le spectre $\mathscr{S}_{\Delta q}(\nu)$ de $\Delta q(t)$ peut également être tracé en coordonnées adimensionnelles :

$$
s=\frac{\nu \Delta}{V} \quad \text { et } \quad \widetilde{\mho I}_{\Delta q}(s)=\frac{\mathscr{S}_{\Delta q}(\nu)}{\Delta p^{2}}\left(\frac{c}{s}\right)^{2} \frac{V}{\Delta}
$$

( $S$ est une section caractéristique des tubes au niveau de la singularité).

Nous avons vu qu'alors $\mathscr{J}_{\Delta q}(s)$ était proportionnel au carré du nombre de Mach $M=V / c$.

Pour les singularités créant une forte zone perturbée à l'aval du circuit, $\widetilde{\Im}_{\Delta g}(s) / M^{2}$ présente un plateau en Strouhal de l'ordre de $10^{-2}$. Les nombres de Strouhal de coupure $s_{1}$ et $s_{2}$ qui limitent ce plateau sont de l'ordre de 0,1 à 0,5 .

Remarque : Les domaines en Strouhal des spectres des fonctions $\Delta p(t)$ et $\Delta q(t)$ étant en général disjoints, on pourra supposer sans faire d'erreur importante que ces fonctions sont décorrélées.

Les ordres de grandeur donnés dans ce paragraphe n'ont qu'une valeur indicative. En effet les paramètres adimensionnels utilisés étant adaptés aux fluctuations locales, les spectres adimensionnels acoustiques sont plus dépendants de la "géométrie" et de la nature des singularités.

\section{Conclusions}

Les études effectuées au CEA/DEMT ainsi que dans d'autres laboratoires, nous ont permis de dresser un premier catalogue de singularités d'écoulement couramment rencontrées dans le circuit.

Les graphiques ci-après, qui en sont tirés, illustrent pour l'élargissement brusque, la façon dont sont présentés les résultats.

Cependant, ce catalogue est loin d'être complet et de nombreuses études tant expérimentales que théoriques sont encore nécessaires pour pouvoir prévoir avec une bonne précision les fluctuations de pression engendrées par les écoulements perturbés dans les circuits, notamment en ce qui concerne des singularités plus complexes ou des singularités couplées très proches les unes des autres.

\section{Elargissement brusque}

1 - Principales grandeurs permanentes de référence

$$
\left(\text { section } S_{1}\right) \stackrel{V}{\rightarrow} \phi D\left(\text { section } S_{2}\right)
$$




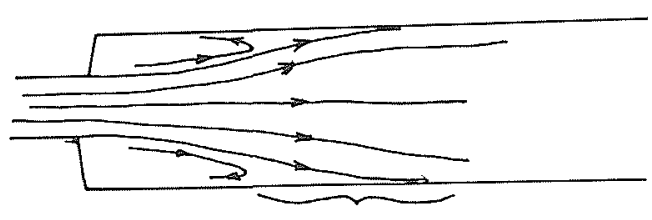

fluctuations maximum

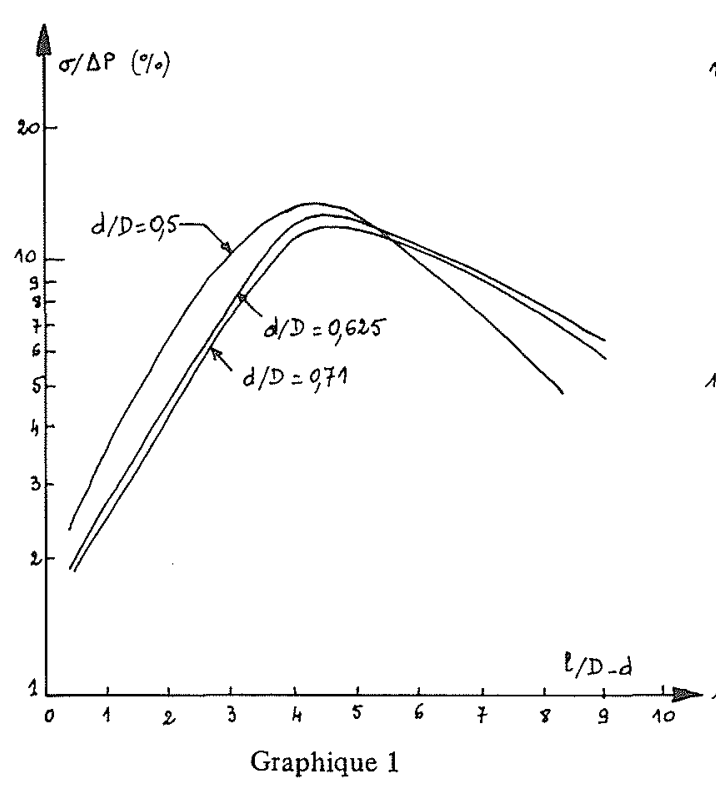

Fig. 2 - Elargissement brusque (fluctuations de pression locales)

Largeur de référence : $D-d$

Paramètre caractéristique $: d / D$

Vitesse de référence : vitesse moyenne du fluide dans le tube amont : $V$

Pression de référence : recompression $\Delta P$ entre l'amont et l'aval.

$$
\Delta P=\frac{Q^{2}}{\rho S_{1} S_{2}}\left(1-\frac{S_{1}}{S_{2}}\right)
$$

$(Q=$ débit masse $)$

2 - Fluctuations locales de pression de paroi (Fig. 2)

- Coordonnées adimensionnelles des spectres

$$
\begin{aligned}
& s=\frac{\nu(D-d)}{V} \\
& F(s)=\frac{\mathscr{S}(\nu)}{\Delta p^{2}} \frac{V}{D-d}
\end{aligned}
$$

- Fonction de cohérence :

$$
C\left(\frac{\Delta \ell}{d}, \Delta \Theta\right) \simeq e^{-0,85}\left|\frac{\Delta \ell}{d}\right|\left|\frac{\Delta \Theta}{\pi / 4}\right|
$$

$(\Delta l=$ distance entre les deux sections droites du tube contenant les deux points de mesure, $\Delta \Theta=$ écart angu-

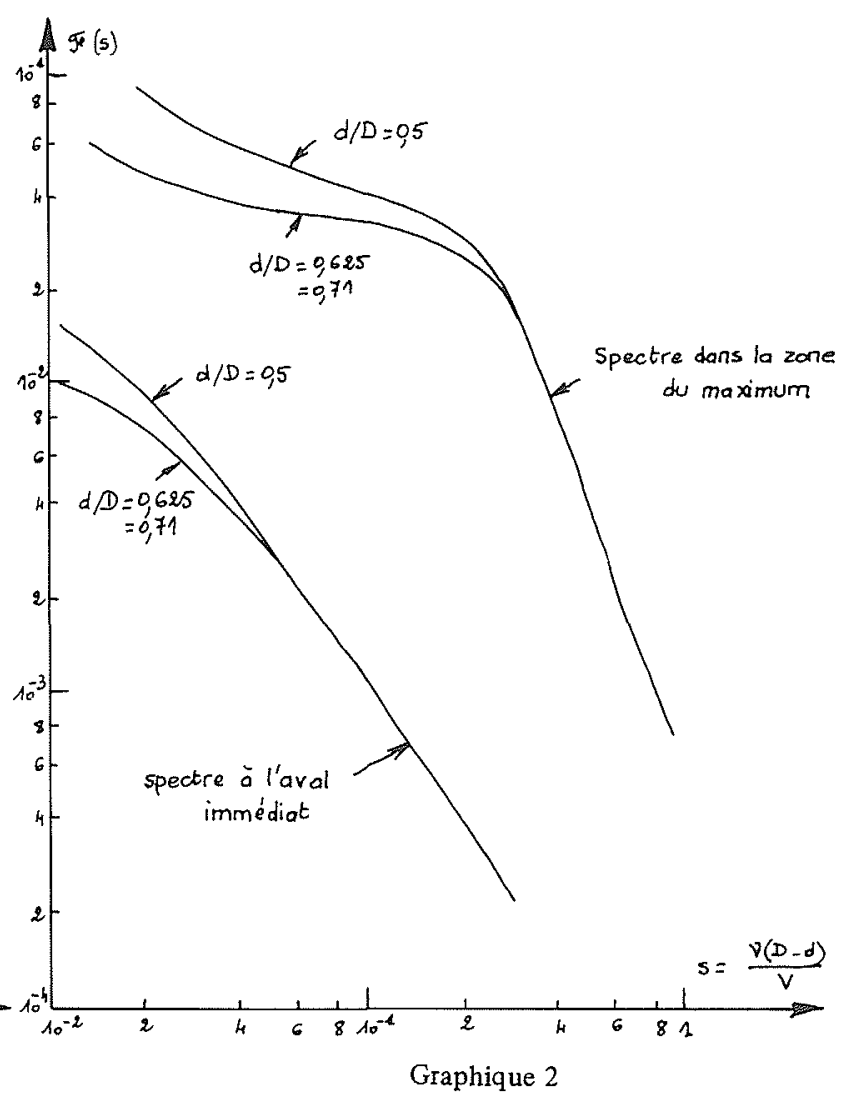

laire azimutal de ces deux points).

Remarque: La cohérence dépend assez peu de la fréquence dans une large plage.

- Fonction phase

$$
\Phi\left(\frac{\nu \Delta \ell}{V}\right) \simeq-\frac{2 \pi}{0,7} \frac{\nu \Delta \ell}{V}
$$

\section{3-Source acoustique associée (Fig. 3)}

- Discontinuité de la pression acoustique :

$$
\widetilde{\mathscr{I}}_{\Delta P}(s)=\frac{\mathscr{S}_{\Delta p}(\nu)}{\Delta P^{2}} \frac{V}{D-d}
$$

- Discontinuité du débit acoustique :

$$
\mathscr{\mathscr { I }}_{\Delta q}(1)=\frac{\mathscr{S}_{\Delta q}(\nu)}{\Delta P^{2}}\left(\frac{c}{s_{2}}\right)^{2} \frac{V}{D-d}
$$

$M=$ nombre de Mach $=\frac{V}{c}$

$c=$ vitesse du son

4-Remarques générales : résultats obtenus pour :

$0,5 \times 10^{5}<R_{e}=\frac{\rho V d}{\mu}<1,5 \times 10^{6}$

$$
\text { et } \quad 0,05<M<0,5
$$




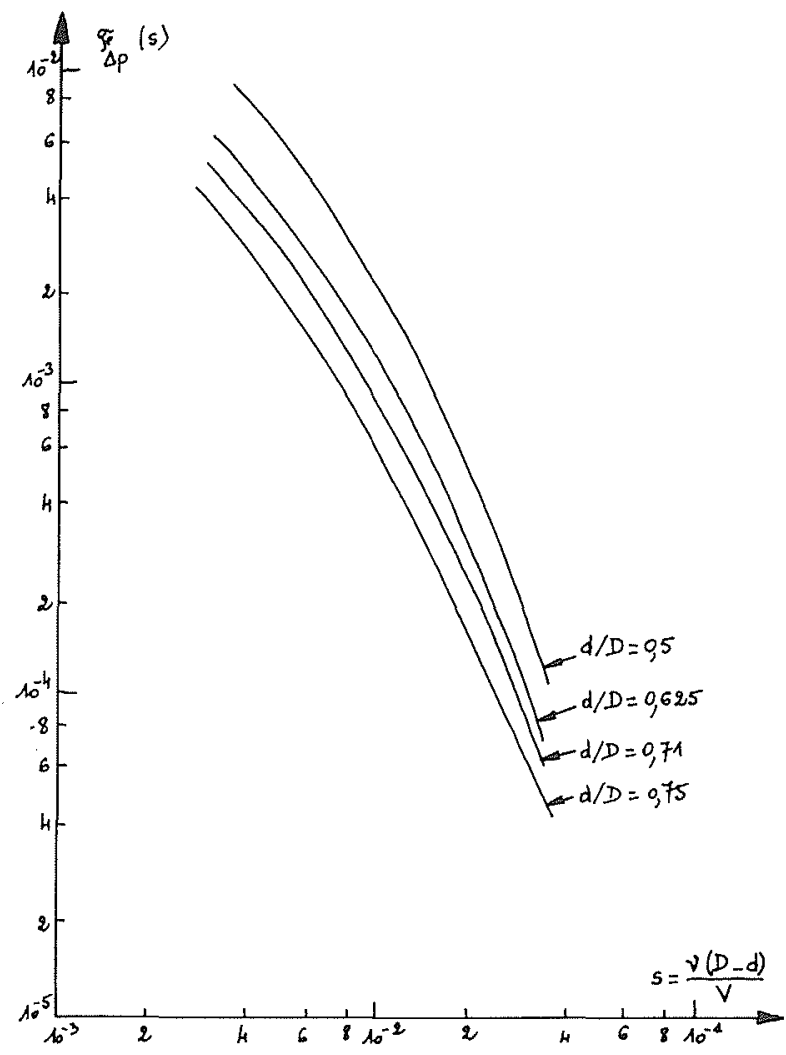

Graphique 3

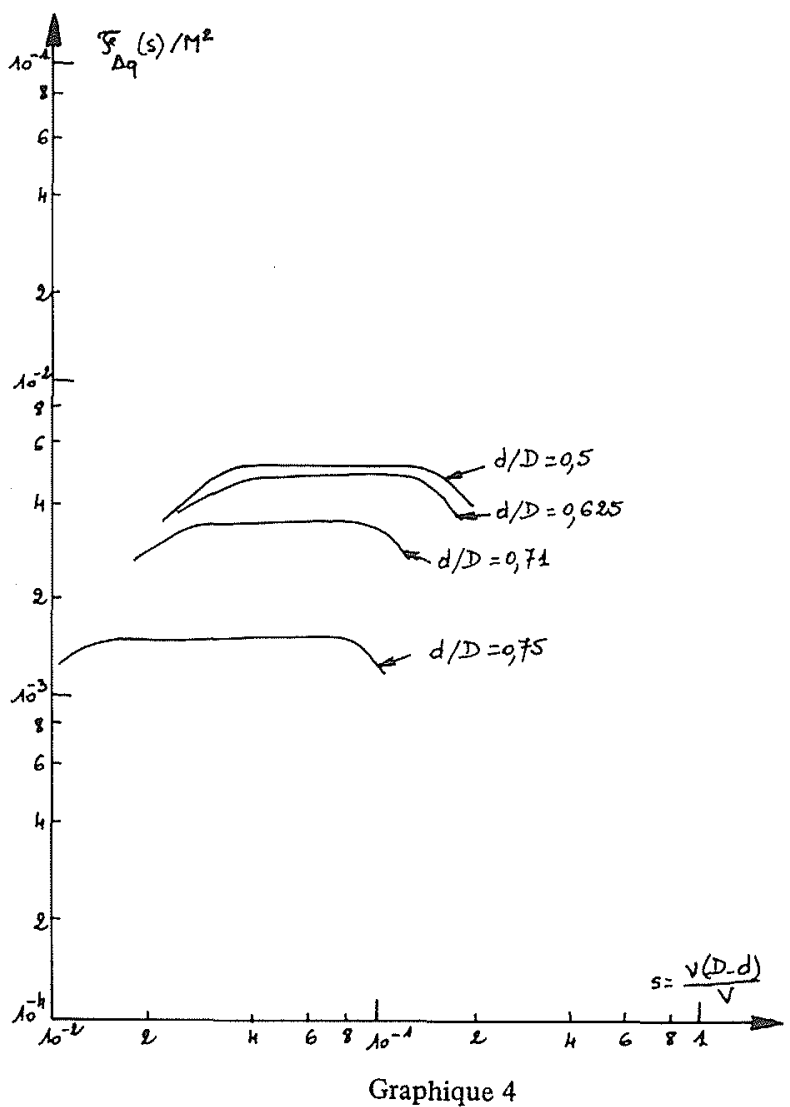

Graphique 4

Fig. 3 - Elargissement brusque (source acoustique)

\section{Références}

[1] LIGHTHILL M.J. - On sound generated aerodynamically, Proc. Roy. Soc., Vol. 211, 1952 - pp. 564-587 and Vol. 224, 1954 - pp. $1-32$.

[2] MORFEY C.L. - Sound transmission and generation in ducts with flow, Int. Of Sound and Vibration Research - Univ. of Southampton March 70.

[3] FORTIER A. - Ecoulements instationnaires dans les circuits, Congrès AIRN 1969.

[4] HENRY R. - Mise en évidence et analyse des instationnarités élastiques dans les écoulements internes, Thèse de Doctorat, Paris VI, 1971.
[5] ROUSE A., ASCE F., JEZDINSKY V. - Fluctuation of pressure in conduit expensions, $J$. Hydraulics Division - Proceeding of the Am. Soc. of Liv. Engineers, 1966.

[6] GIBERT R.J. - Etudes expérimentales de deux singularités d'un circuit, Note CEA - 1735 - 1974.

[7]GIBERT R.J., LIVOLANT M., BAYLAC G. - Calcul de l'amplification acoustique dans les circuits complexes, Lo Houille Blanche, $\mathrm{n}^{\circ} 5-1971$.

[8] GIBERT R.J., VILLARD B. - Sources acoustiques associées à la réunion de deux écoulements dans un circuit, Congrès FA.S.S.E. 75 , Oct. 75 .

[9] GIBERT R.J. - Etude des fluctuations de pression dans les circuits parcourus par des fluides, Note CEA $n^{\circ} 1925,1976$. 


\section{Discussion}

Président : M. J.PAULON

M. le Président. - Je remercie M. GIBERT pour son intéressant exposé et j'ouvre la discussion.

M. FANELLI. - Est-ce qu'on a essayé de mettre la même singularité dans deux circuits nettement différents au point de vue réponse acoustique et de vérifier si les caractéristiques d'excitation de la source sont les mêmes dans les deux cas?

M. GIBERT. - Personnellement, j'ai étudié un élargissement brusque, $d$ 'abord en air dans une veine d'essai comprise entre une aspiration atmosphérique et un col sonique, ensuite dans un circuit en eau compris entre deux bidons de tranquillisateur. Les résultats se recoupent très bien.

M. FANELLI. - Etes-vous d'accord qu'en principe on devrait faire des recherches pour vérifier s'il y a des réactions des caractéristiques du circuit sur les distributions spectrales de la source?

M. GIBERT. - Dans la plupart des cas qu'on a considérés, on n'a pas observé d'interaction ; mais on a observé quand même certains cas de couplage. En général, ces couplages ont lieu sur des fréquences particulières.

Je peux citer le cas d'un diffuseur qu'on a réalisé d'une façon incorrecte. En fait, il y avait un mauvais raccord entre tuyauteries tout près du diffuseur au niveau d'une bride. Le tube amont n'avait pas tout-à-fait le même diamètre que le tube aval ; il y avait donc une sorte de décrochement.

Là, on a observé, pour une certaine plage de vitesse d'écoulement, un phénomène acoustique très intense à une fréquence bien déterminée et cette fréquence correspondait au premier mode en demi-onde du tube amont.

En pareil cas, il y a visiblement une interaction entre l'onde et l'écoulement fluctuant. C'est quelque chose qu'on n'a pas analysé finement. Mais on a constaté que lorsqu'on a supprimé ce petit décrochement, le phénomène a disparu. Il y avait peut être une extrême sensibilité de l'écoulement à ce niveau à une variation de la pression.
M. FANELLI. - Quand il y a deux singularités proches l'une de l'autre cela doit se produire davantage?

M. GIBERT. - Peut-être. Des études ont été menées par BERTIN sur l'interaction de coudes : deux coudes étaient placés l'un derrière l'autre dans différentes configurations. On n'a pas relevé de phénomènes d'interaction très importants dans ce cas.

M. MILAN. - En ce qui concerne la réponse du circuit à ces sollicitations dynamiques, comment peut-on faire la part des choses entre le phénomène local et le phénomène acoustique global?

M. GIBERT. - Les circuits sont excités par les écoulements fluctuants sur leurs premiers modes propres. Ces modes ont des déformées évoluant assez lentement spatialement.

Ainsi, les dimensions des zones perturbées associées aux singularités sont suffisamment faibles, pour que l'on puisse supposer un simple mouvement de translation des structures dans ces zones.

La force généralisée (projection et efforts sur la déformée modale) peut donc être confondue avec la résultante générale des efforts locaux au niveau de la singularité.

Or, on peut montrer que cette résultante générale ne dépend que de la pression dans le fluide à l'amont et à l'aval de la singularité, et donc que le problème se ramène à un problème acoustique.

Il est alors facile de montrer que l'ensemble des efforts d'excitation de la singularité peut être représenté par la discontinuité de la pression acoustique dont nous avons parlé dans l'exposé.

Ces considérations ont été vérifiées expérimentalement sur une tuyauterie en écoulement d'eau excitée par une vanne à demi-fermée. 